\title{
Principles of fluid mechanics applied to some situations in the human circulation and particularly to the testing of valves in a pulse duplicator
}

\author{
LESLIE J TEMPLE, R. SERAFIN, N. G. CALVERT, \\ AND JEAN M. DRABBLE \\ From the Departments of Surgery and Engineering, University of Liverpool, and the \\ Regional Cardiac Centre of the Liverpool Regional Hospital Board
}

The use of a pulse duplicator to test prosthetic aortic valves or to investigate the mechanics of diseased valves after death is now well established (McMillan, Daley, and Matthews, 1952; McMillan, 1955).

In testing an artificial valve, two main questions have to be answered. The first concerns the mechanical strength of the valve and therefore its ability to withstand for many years pressures and flow rates comparable to those it would experience in the human body. The second problem is to determine whether the flow characteristics of the prosthetic valve are suitable for clinical use. Until now the pulse duplicator has been used to answer only the first question and little attention has been paid to the second.

In preliminary experiments with a new type of pulse duplicator, we experienced some difficulty in producing simultaneously with water a pressure wave form and quantity flow comparable to those produced by blood in life. So far as the authors are aware, almost all pulse duplicators hitherto reported use water as a working fluid (Davila, Trout, Sunner, and Glover, 1956; Kelley, Goodale, and Castleman, 1960; Callaghan, Willans, and Cardozo, 1961). The use of glycerinewater mixtures, as reported by Marx, Baldwin, and Kittle (1959) and Starkey, Sirak, Collins and Hagan (1963), is exceptional. There are sound practical reasons for this, but the significance of the results when using water instead of blood is open to criticism. Blood is more viscous than water, and it seems that it should be easier to perfuse water through a heart than blood. In practice, the reverse appeared to be true. This difficulty forced on us the more fundamental examination of the problem, which is the subject of this paper.

\section{TYPES OF FLUID FLOW}

In order to make clear the arguments that follow, it is useful to look first at some of the flow phenomena that may occur in the circulation.

Fluid flow in a closed duct may be either laminar or turbulent. The properties of flow change markedly between these two states. In changing from one type of flow to the other, there is normally an unstable transition period.

LAMINAR FLOW The characteristic of laminar flow is the absence of lateral mixing. A stream of dye introduced into laminar flow remains distinct. The liquid in fact moves in a series of concentric cylinders like a well lubricated expanding telescope, minimizing friction both against the walls of the duct and between the particles of fluid themselves. Any disturbance introduced into such a flow will be damped out.

TURBULENT FLOW In turbulent flow, although there is a general forward movement, particles are moving in all directions. There is no smooth sliding of layers against one another, and the turbulence in itself acts as an obstruction so that a greater force is required to move the body of fluid onwards. Dye introduced into the stream spreads and diffuses throughout the fluid.

BOUNDARY LAYER In a flow past a solid surface there is always a layer of fluid across which the velocity must change from zero at the stationary surface to the main stream velocity. This region is called the boundary layer and is generally, though not always, thin compared with the dimensions of the flow. 
Direct observations of flow in small blood vessels near the surface have shown a plasma boundary layer surrounding a core of whole blood (Taylor, 1959). The same kind of separation probably occurs also in the larger vessels of the body. This has never been confirmed experimentally, but there is a theoretical basis for believing it. This theory is supported by evidence from studies of the pumping of coal suspensions and paper stock (Wilkinson, 1960).

Because plasma is less viscous than whole blood (Merrill and Wells, 1961), the existence of such a plasma boundary layer would promote laminarity at flow rates at which homogenous fluid would tend to break down into turbulence.

EFFECTS OF ACCELERATION It is well established that accelerated flow tends towards stability and laminarity, and retarded flow towards instability and turbulence. 'Accelerated flow' may mean either that the velocity at a particular point is increasing with time, or that a particular particle is moving into a region of higher velocity, as, for example, when flowing through a converging passage.

BOUNDARY LAYER CONTROL BY THE CORONARY ARTERIES When an initially laminar flow is stopped, a forward motion continues in the core of the pipe for a considerable time, being balanced by an equal return flow near the walls (Calvert and Drabble, 1962).

In a simple tube, this return flow would circulate back into the core, possibly producing turbulence (Fig. 1). If, however, it was drawn off before re-entering the core, turbulence from this source would be prevented. This is a form of the technique known as 'boundary layer control'.

When the aortic valve closes at the end of systole there is an abrupt cessation in the accelerated

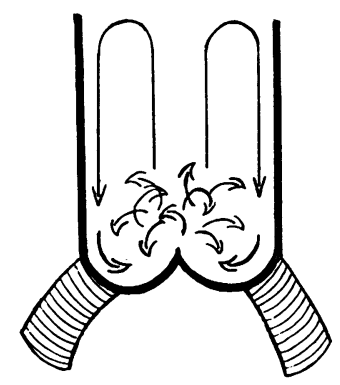

FIG. 1. The aorta as a simple tube without coronary arteries showing the return flow at the end of systole breaking into the central core to cause turbulence.

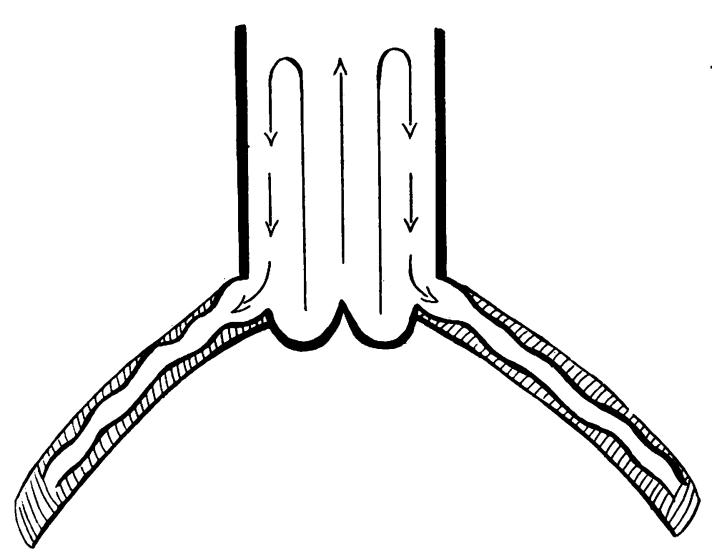

FIG. 2. Coronary arteries and aorta showing draining off of return flow as a form of boundary layer control.

flow along the aorta. In these circumstances it $\vec{\bullet}$ appears that the filling of the coronary arteries in $\mathscr{O}$ diastole provides a boundary layer control. In all other situations in the body, branches of main arteries, even if destined eventually to run backwards against the main stream, take off initially at an acute angle to the direction of flow. Only the coronary arteries branch off against the direc- $\mathbb{\Phi}$ tion of the main stream. The direction of the $\overrightarrow{\overrightarrow{0}}$ coronary arteries ensures that the reversed boun- 3 dary layer fills them efficiently in diastole (Fig. 2). Since this returning layer is a spill-over from the core, it will contain a normal proportion of cells and plasma. It is appreciated that compression of $\mathrm{O}$ the coronary arteries by the contracting ventri- $\tilde{x}$ cular muscle prevents flow in them during the



FIG. 3. Left ventricle and aorta in systole showing that occlusion of the coronary arteries by contraction of the muscle prevents venturi effect. 
systole but also prevents the sucking out of blood from the coronaries by the venturi effect that would otherwise occur when blood moves forward past their orifices (Fig. 3).

\section{EXPERIMENTAL DATA}

In order to demonstrate the presence of turbulence or laminarity, human hearts with normal aortic valves were used. Into the stump of the aorta $1 \mathrm{~cm}$. above the valve, a cylinder of glass $2.5 \mathrm{~cm}$. in diameter and $20 \mathrm{~cm}$. in length was tied. To the other end of the glass tube an artificial resistance was attached. Water was pumped into the left ventricle, either continuously or in a pulsatile manner resembling the normal cardiac cycle. A fine hypodermic needle was inserted through the base of the aorta, and through this a jet of India ink was injected automatically in each diastole (Fig. 4). The rate of flow was varied by adjusting the inflow pressure to the left ventricle.

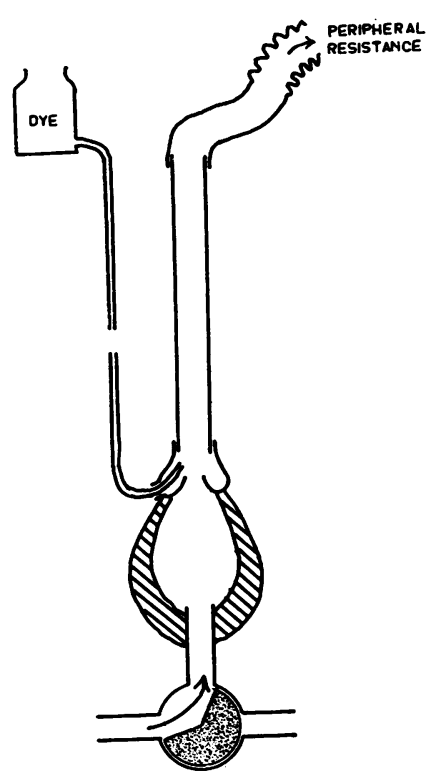

FIG. 4. The arrangement for studying turbulence or laminarity in the aorta. Perfusion of the left ventricle with automatic injection of dye in each diastole.

Each injection of India ink took on a bizarre but quite sharply angular shape lying in the surrounding clear fluid. Laminarity was demonstrated by the movement of this mass of dye up the glass tube during subsequent systole without a significant change in shape. At low flow rates the dye mass could be followed for two systoles, and still it retained its clearly defined shape, demon-
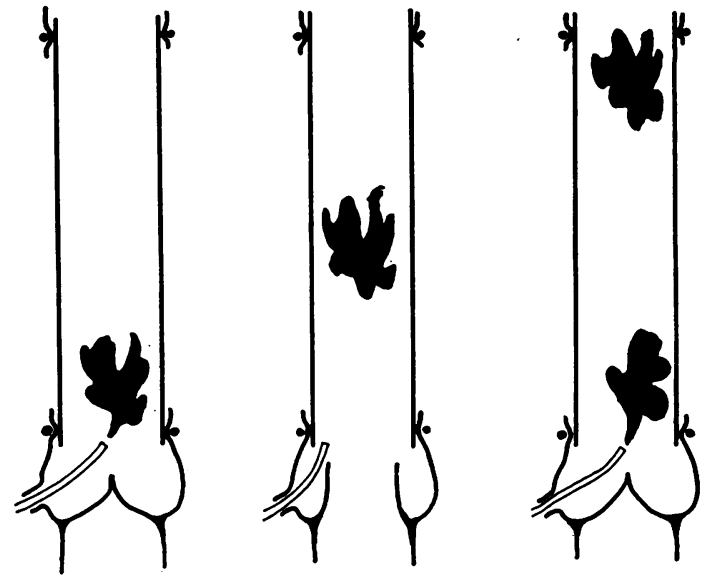

FIG. 5. Successive pictures of dye injected above a normal aortic valve with the use of a pulse duplicator. Laminar flow allows the mass to retain its shape.

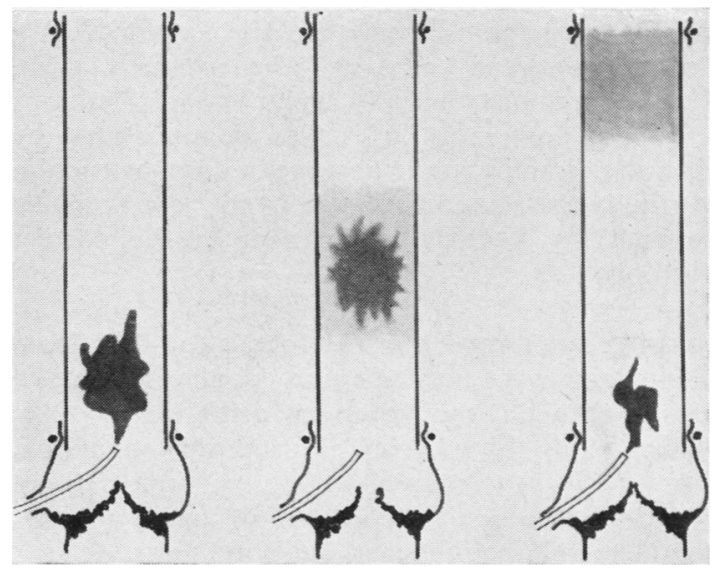

FIG. 6. The aorta above a calcified stenosed aortic valve showing break-up of the dye in turbulence.

strating a complete lack of mixing (Fig. 5). Turbulence was indicated when, following systole, the dye spread uniformly throughout the moving fluid, no dye mass being recognizable (Fig. 6).

Whereas laminarity and complete turbulence are unmistakable, when conditions were changing from one to the other there was always an intermediate stage when partial mixing of the dye occurred. All these changes could readily be recorded by slow-motion cine-photography, but still photographs of the rapidly moving dye proved beyond our technical resources.

CONTINUOUS PERFUSION OF NORMAL HEARTS Flows up to $1 \frac{1}{2}$ litres $/ \mathrm{min}$. produced laminarity. Flows over 2 litres $/ \mathrm{min}$. produced turbulence. 
PULSATILE FLOW THROUgh NORMAL HEARTS At pulse rates between 25 and $70 / \mathrm{min}$., flows up to 6 litres $/ \mathrm{min}$. produced laminarity. Flows over 8 litres $/ \mathrm{min}$. produced turbulence.

PULSATILE FLOW THROUGH HEARTS WITH TIGHT AORTIC STENOSIS Even at the lowest flow rates that were technically feasible, i.e., below $1 \frac{1}{2}$ litres/ min., gross turbulence occurred.

\section{CLINICAL EXAMPLES}

In order to relate our findings on the dead heart to conditions in life we have looked for evidence of turbulent and laminar flow in health and disease.

STREAMING The phenomenon of 'streaming' during cardiac catheterization, in which widely different oxygen values are found in the right atrium and ventricle, is taken by cardiologists in the absence of any shunt to be due to a failure to mix of the streams from the superior vena cava, the inferior vena cava, and the coronary sinus. This is laminarity usually under basal conditions with low flow rates. It is also demonstrated by cine-angiography in the absence of any obstruction to the vessels outlined, when the dye can be 'layered' by keeping the patient in the correct position.

FOETAL CIRCULATION In the foetus, the flow from the inferior vena cava carrying the placental blood passes through the foramen ovale to the left atrium whilst blood from the superior vena cava crosses the same cavity to the tricuspid valves. We think that only laminar flow could prevent complete mixing under these conditions.

VESSEL DAMAGE The effect of turbulence on the vessel wall could be anticipated from observation

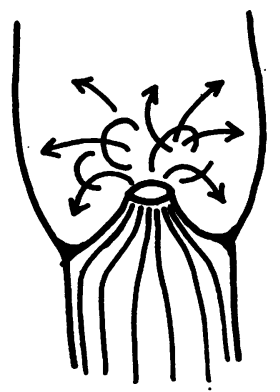

FIG. 7. Laminar flow in a great vessel being accelerated as it passes through a stenosed valve, giving rise to turbulence beyond the stenosis. of the turbulent dye particles striking the walls in all directions. With normal cardiac output, the velocity of blood flow through a stenotic valve opening into a normal vessel produces severe turbulence just beyond the stenosis, as shown in our experiments (Fig. 7).

The paradox of a post-stenotic dilatation of a pulmonary artery or aorta (in which the pressure is lower than normal) can be explained by the turbulent movement of the blood striking the walls of the artery like a pneumatic hammer instead of gliding in well lubricated layers over it. The aorta above a coarctation retains its contour under high pressure, but beyond the constriction the low pressure segment is dilated. These observations could be best explained by laminar flow above the coarctation and turbulence below it.

Normal branching of vessels does not cause turbulence in the dividing stream, but entry of blood into a stream against the direction of flow is calculated to produce local turbulence. Such a condition is found in patent ductus arteriosus and in the entry of intercostal arteries carrying a collateral blood flow into the aorta below a coarctation. In both these sites infective endarteritis and mycotic aneurysms are found, the site of infection being determined by damage to the vessel wall.

VALVE DAMAGE The chordae tendineae of the mitral valve in mitral stenosis continue to shrink and thicken long after rheumatic activity has passed. This change is perhaps most readily illustrated by restenosis after incomplete valvotomy. Repeated minor damage and the deposition of fibrin from turbulent blood issuing through the narrowed orifice might well contribute to this.

BLOOD DAMAGE The damaging effect of turbulence on red blood cells is perhaps a little more speculative. We have already referred to the boundary layer of plasma that normally protects the red cells from contact with the vessel wall. In turbulent o flow this layer could be scoured away, allowing. N the red cells to come into collision with the wall. $N$ This could produce both haemolysis and clotting. That in fact this does occur is shown by the laminated clot deposited by the swirling turbulence of the blood in an aneurysm and the clotting and haemolysis that are known to have occurred in $\square$ some of the earlier models of prosthetic aortic $\overline{0}$ valves. The best documented instances of blood: $\mathbb{D}$ damage have occurred when a jet of blood pro- $\frac{\Omega}{\mathbb{D}}$ duced turbulence in the region of a teflon intracardiac patch (Sayed, Davie, Hendley, Lewis, and Cleland, 1961). 
Anaemia in patients with valvular heart disease is commonly recognized, but there are few indications as to its cause, although haemolysis from turbulence might well be one. The only indication that this is so is the frequent finding of a positive Coombs' test in such patients.

INFERENCE We conclude that in the normal circulation laminarity is the rule and turbulence is associated with pathological changes.

\section{THEORETICAL CONSIDERATIONS}

We shall demonstrate that the flow characteristics of a system such as the human great vessels can be defined in terms of two parameters based on the known constants within the system. The first parameter relates to both continuous and intermittent flow and is known as Reynolds number. The second is concerned specifically with pulsatile flow and is dependent on the pulse rate. In two different fluid flow systems of the same geometrical shape, if each of the two parameters described is the same in both systems, then the flow characteristics will be identical. If flow is turbulent or laminar in one system, it will be the same in the other. This is the principle of dynamic similarity. Only by the use of this principle is it possible to interpret experimental data in terms of living physiology.

APPLICATION OF THE PRINCIPLE OF DYNAMIC SIMILARITY TO THE PULSE DUPLICATOR. The principle of dynamic similarity states that if a physical problem is governed by $\mathbf{N}$ independent variables, each of which can be expressed in terms of the three dimensions, mass, length, and time, then the relation between these variables can equally be expressed as a relation between $(\mathrm{N}-3)$ dimensionless parameters formed from them. Two systems will be 'dynamically similar' provided each of these dimensionless parameters is the same for both, even though the individual variables may be different.

The significant independent variables of the pulse duplicator are considered to be those listed below. We assume that the wave form of the machine is similar to that in life, and that the important parts of the system are the same shape as in life.

From the following five variables, $p=$ the density of the operating fluid; $\mu=$ the viscosity of the operating fluid; $Q=$ the flow rate; $d=a$ representative dimension, which may be taken as the diameter of the aorta; and $\mathrm{f}=$ the pulse rate, we can form two dimensionless parameters. These could be

$$
\frac{\rho Q}{\mu d} \text { and } d \sqrt{\frac{f \rho}{\mu}}
$$

The first of these (usually expressed as $\frac{4 \rho Q}{\pi \mu d}$ ) is called Reynolds number. The second, when multiplied by $\frac{\pi}{\sqrt{ } 2}$ is the parameter ' $\alpha$ ' used to describe pulsatile flows (Cotton, 1960).

If a dead heart is perfused with water the flow properties will be the same as in life if, and only if, both these parameters have the same values in the pulse duplicator as in life. The practical consequences of these requirements are shown as follows:

The suffix $w$ refers to conditions using water in the pulse duplicator, and suffix $b$ to blood flow in life.

For dynamically similar conditions, we require first that the Reynolds numbers are the same:

$$
\frac{\rho_{\mathrm{w}} \mathrm{Q}_{\mathrm{w}}}{\mu_{\mathrm{w}} \mathrm{d}_{\mathrm{w}}}=\frac{\rho_{\mathrm{b}} \mathrm{Q}_{\mathrm{b}}}{\mu_{\mathrm{b}} \mathrm{d}_{\mathrm{b}}}
$$

If we take the density of blood as approximately equal to that of water $\left(\rho_{\mathrm{w}}=\rho_{\mathrm{b}}\right)$, and if the live and dead aortas are the same size $\left(d_{w}^{d}=d_{t}\right)$, then

$$
\frac{\mathrm{Q}_{\mathrm{w}}}{\mathrm{Q}_{\mathrm{b}}}=\frac{\mu_{\mathrm{w}}}{\mu_{\mathrm{b}}}
$$

Secondly, the values of $\alpha$ must be the same:

$$
d_{w} \sqrt{\frac{f_{w} \rho_{w}}{\mu_{w}}}=d_{b} \sqrt{\frac{f_{b} \rho_{b}}{\mu_{b}}}
$$

Again putting $\mathrm{d}_{\mathrm{w}}=\mathrm{d}_{\mathrm{b}}$, we obtain

$$
\frac{\mathrm{f}_{\mathrm{w}}}{\mathrm{f}_{\mathrm{b}}}=\frac{\mu_{\mathrm{w}}}{\mu_{\mathrm{b}}}
$$

Thus, in words, if we wish to achieve conditions in the pulse duplicator similar to those obtaining in life, we must reduce both the flow rate and the pulse rate in the ratio of the viscosities of blood and water.

The principle also states that any dimensionless parameter containing one dependent variable will have the same value in both systems when dynamically similar conditions prevail. Thus, for example, if a certain pressure gradient $\triangle p$ depends only on the flow conditions, then the parameter $\frac{\Delta p \cdot d^{4}}{\rho Q^{2}}$, which is dimensionless, will be a constant if both the Reynolds number and the pulse rate parameter are constants.

$$
\begin{array}{ll}
\text { Thus } & \frac{\Delta \mathrm{p}_{\mathrm{w}} \mathrm{d}_{\mathrm{w}}{ }^{4}}{\rho_{\mathrm{w}} \mathrm{Q}_{\mathrm{w}}{ }^{2}}=\frac{\Delta \mathrm{p}_{\mathrm{b}} \mathrm{d}_{\mathrm{b}}{ }^{4}}{\rho_{\mathrm{b}} \mathrm{Q}_{\mathrm{b}}{ }^{2}} \\
\text { or } & \frac{\Delta \mathrm{p}_{\mathrm{w}}}{\Delta \mathrm{p}_{\mathrm{b}}}=\frac{\mathrm{Q}_{\mathrm{w}}{ }^{2}}{\mathrm{Q}_{\mathrm{b}}{ }^{2}}=\frac{\mu_{\mathrm{w}}{ }^{2}}{\mu_{\mathrm{b}}{ }^{2}}
\end{array}
$$

Hence the pressure gradient in the pulse duplicator will be smaller than the corresponding value in life in proportion to the square of the viscosity ratio.

The application of these results is illustrated by the following numerical example:

Viscosity of blood at $36^{\circ} \mathrm{C} .=\mu_{\mathrm{b}}=4.0$ centipoise

Viscosity of water at $15^{\circ} \mathrm{C} .=\mu_{\mathrm{w}}=1.14$ centipoise 
Hence viscosity ratio $=\frac{\mu_{\mathrm{w}}}{\mu_{\mathrm{b}}}=\frac{1}{3 \cdot 5}$

Thus if blood flow in life $=Q_{b}=5$ litres $/$ min., then required water flow $=Q_{w}=\frac{5}{3.5}=1.43$ litres $/ \mathrm{min}$.

Also if pulse rate in life $=f_{b}=84$ per minute, then required pulse rate in duplicator $=f_{w}=\frac{84}{3 \cdot 5}=24$ per minute.

If a pressure gradient $\Delta \mathrm{p}_{\mathrm{w}}$ of $1 \mathrm{~mm}$. of mercury is measured on the pulse duplicator, the corresponding pressure gradient in life $\Delta p_{b}$ will be $1 \times 3.5^{2}=$ $12 \cdot 25 \mathrm{~mm}$. mercury.

EFFECT OF USING WATER INSTEAD OF BLOOD IN A PULSE DUPLICATOR If the Reynolds number in the aorta rises above a certain level, the flow will normally be turbulent ; if it is below another figure it is always laminar. Between these two values there is a transitional phase when flow conditions cannot be forecast with certainty. Since, as we have shown, the Reynolds number is inversely proportional to the viscosity, the use of water instead of blood in perfusing the heart can result in the conversion of a normal laminar flow to turbulence unless the flow velocity is reduced in the ratio of the two viscosities, i.e., 3.5 to 1 , to maintain similar conditions.

It is this factor that explains our initial difficulty in obtaining high flow rates with water pumped through the heart.

The pressure gradient across a valve can be measured on the pulse duplicator and theoretically should be multiplied by 12.25 to obtain the corresponding figure for the circulation of blood. In fact, this is highly inaccurate. Small differences in diameter between the glass tube and the aorta will lead to errors in calculating flow rates that are acceptable. If, however, pressure gradients are calculated, the errors are squared and become significant. Further refinements of technique are needed before pressure gradients can be reliably predicted.

Attempts to use the figures obtained on a pulse duplicator as if they would apply in life (Lillehei, 1963) will lead to difficulty.

\section{DISCUSSION}

The application of the principle of dynamic similarity to the pulse duplicator allows us to interpret our experimental findings in terms of the human circulation. We found that turbulence occurred at a perfusion rate of 8 litres of water per minute through the ascending aorta. This $\overrightarrow{\vec{S}}$ corresponds to perfusion of 28 litres of blood per $\overline{0}$ minute. To relate this to total cardiac output we 음 have to add the amount of blood that would pass through the coronary arteries during similar flows. This figure has never been determined (Best and Taylor, 1961), but proportions of up to $30 \%$ have been recovered from the coronary sinus during by-pass surgery. This suggests that turbulence will occur in the human aorta when cardiac output reaches between 30 and 40 litres per minute.

This figure fails to take account of two factors we have discussed. The plasma boundary layer facilitates greater velocity without turbulence, and the mechanism of boundary layer control by the coronaries that we have postulated allows even greater flows in laminar conditions.

Two other factors may have an influence. Whereas turbulence is directly related to the Reynolds number, no such relationship has been shown for the parameter that is concerned with the pulse rate, and nothing in our work has shown such a relationship. However, since our experimental work was carried out at rates that allowed dynamic similarity, the present interpretation is valid. The other factor of unknown value depends on the anomalous viscosity of blood, which varies with the shear rate, i.e., the rate at which layers $\overline{\vec{z}}$ of fluid slide on one another (Merrill and Wells, 1961). The value used in our calculations is that $\supset$ approached at an infinite shear rate. This was shown (Taylor, 1959) to be the most suitable value for the pulsatile conditions obtaining in the aorta.

It is clear to us that the calculated level at $\underset{x}{x}$ which turbulence appears in the aorta approxi- $\dot{\sigma}$ mates very closely to the known maximum cardiac 3 . output in exercise. This fits the fact that the $\delta$ presence of turbulence would lead to a dispropor- 3 tionate increase in cardiac work to maintain the 을 output and that outputs above this level are therefore unphysiological.

We can deduce from our experiments with hearts from patients who had died with aortic $\sigma$ stenosis that turbulence appeared before the $N$ cardiac output had reached $20 \%$ of normal $\underset{\omega}{N}$ maximum, and possibly at much lower levels than 0 our apparatus could simulate.

\section{CONCLUSION}

In normal health we think turbulence does not occur in the circulation even at the extremes of exercise. Our experimental data pointing to this $\mathbb{\mathbb { }}$ are reinforced by our clinical observations that laminarity is the rule in the normal circulation, 
and turbulence, when it occurs, is associated with pathological changes.

Any operation on or replacement of a cardiac valve should aim at restoring normal physiological conditions. We have shown that the stenosed valve readily gives rise to turbulence. To replace it with a prosthesis which, although overcoming the stenosis, causes turbulence at rest or on exercise because of its shape or structure, is ineffective. The turbulence itself will add to the burden of an already damaged ventricle.

A prosthetic valve may fail because it fails to deal with the additional biological problems, but it cannot succeed unless it at least satisfies the simple engineering concepts that are accepted for all fluid flow systems. If a human organ is perfused with a fluid other than blood, it must satisfy the known engineering problems as well as the often unknown physiological ones if valid observations are to be made.

We therefore conclude that the testing of prosthetic valves must include the investigation of their flow characteristics under conditions dynamically similar to those in life, including exercise. The technique described in this paper appears to be adequate for this purpose.

\section{SUMMARY}

The application of the principles of fluid mechanics to the water perfusion of dead hearts indicates that the current practice in testing prosthetic valves fails to evaluate them under conditions of flow comparable to those in life.

Flow patterns and the properties which depend upon them, such as turbulence and the pressure drop across a restriction, can only be studied with water under conditions approaching dynamic similarity. In practice, this means reducing the flow rate and pulse rate in the ratio of the viscosities of blood and water (about $1: 3 \frac{1}{2}$ ). The presence of turbulence in these conditions can be detected by the injection of dye. The vastly greater efficiency of pulsatile flow compared to steady flow as shown by laminarity at high flow rates has been demonstrated.

Attention is drawn to the known adverse effects of turbulence in the blood stream and the importance of selecting prosthetic valves that allow laminar flow.

The authors gratefully acknowledge the assistance and encouragement of Professors J. H. Horlock and J. H. Preston of the Faculty of Engineering of the University of Liverpool. The expenses of this research were borne by the Medical Research Committee of the United Liverpool Hospitals, and one of us (R. S.) was in receipt of a grant from that Committee.

\section{REFERENCES}

Best, C. H., and Taylor, N. B. (1961). The Physiological Basis of Medical Practice, 7th ed. p. 321, Bailliè re, Tindall and Cox, London.

Callaghan, J. C., Willans, M. J., and Cardozo, D. (1961). In Prosthetic Valves for Cardiac Surgery, ed. K. A. Merendino, p. 48. Thomas, Springfield, Ill.

Calvert, N. G., and Drabble, J. M. (1962). In Proceedings National Engineering Laboratory Symposium on Flow Measurement in Engineering Laboratory Symposium on Flow Measurem
Closed Conduits, Vol. 2, p. 667 . H.M.S.O., Edinburgh.

Cotton, K. (1960). Instantaneous measurement of velocity of blood flow and of vascular impedance. Ph.D. thesis, London.

Davila, J. C., Trout, R. G., Sunner, J. E., and Glover, R. P. (1956). A simple mechanical pulse duplicator for cinematography of cardiac valves in action. Ann. Surg., 143, 544.

Kelley, R. R., Goodale, F., and Castleman, B. (1960). The dynamics of rheumatic and calcific aortic valve disease. A post-mort $\mathrm{\epsilon m}$ study with the use of the pulse duplicator. Circulation, 22, 365 .

study with the use of the pulse duplicator. Circulation, 22, 365.
Lillehei, C. W. (1963). Discussion. J. thorac. cardiovasc. Surg., 45, 62 .

Marx, T. I., Baldwin, B. R., and Kittle, C. F. (1959). A cardiovascular simulator for the evaluation of prosthetic aortic valves. Ibid., 38, 412 .

McMillan, I. K. R. (1955). Aortic stenosis. A post-mortem cinephotographic study of valve action. Brit. Heart J., 17, 56.

Daley, R., and Matthews, M. B. (1952). The movement of aortic and pulmonary valves studied post mortem by colour cineand pulmonary valves studic
matography. Ibid., 14, 42 .

Merrill, E. W., and Wells, R. E., Jr. (1961). Flow properties of biological fluids. Appl. Mech. Rev., 14, 663.

Sayed, H. M., Davie, J. V., Handley, D. A., Lewis, S. M., and Cleland, W. P. (1961). Haemolytic anaemia of mechanical origin after open heart surgery. Thorax, 16, 356.

Starkey, W. L., Sirak, H. D., Collins, J. A., and Hagan, B. T. (1963). The design and development of a cardiac simulator for the evaluation of heart-valve prosthesis. J. thorac. cardiovasc. Surg., 46, 207.

Taylor, M. G. (1959). The influence of the anomalous viscosity of blood upon its oscillatory flow. Phys. in Med. Biol., 3, 273.

Wilkinson, W. L. (1960). Non-Newtonian Fluids. p. 128. Pergamon Press, London, 